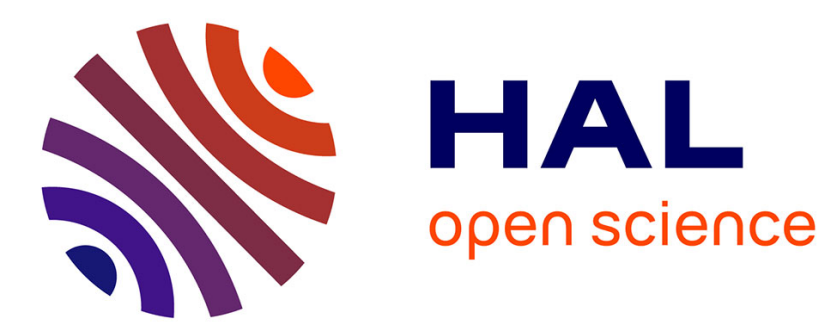

\title{
Control Allocation of Active Rear Steering and Vehicle Dynamics Control Using a New Tire Model
}

\author{
Moad Kissai, Bruno Monsuez, Adriana Tapus, Didier Martinez
}

\section{To cite this version:}

Moad Kissai, Bruno Monsuez, Adriana Tapus, Didier Martinez. Control Allocation of Active Rear Steering and Vehicle Dynamics Control Using a New Tire Model. International Journal of Mechanical Engineering and Robotics Research, 2018. hal-02002718

\section{HAL Id: hal-02002718 https://hal.science/hal-02002718}

Submitted on 31 Jan 2019

HAL is a multi-disciplinary open access archive for the deposit and dissemination of scientific research documents, whether they are published or not. The documents may come from teaching and research institutions in France or abroad, or from public or private research centers.
L'archive ouverte pluridisciplinaire HAL, est destinée au dépôt et à la diffusion de documents scientifiques de niveau recherche, publiés ou non, émanant des établissements d'enseignement et de recherche français ou étrangers, des laboratoires publics ou privés. 


\title{
Control Allocation of Active Rear Steering and Vehicle Dynamics Control Using a New Tire Model
}

\author{
Moad Kissai, Bruno Monsuez, Adriana Tapus \\ ENSTA ParisTech / Department of Computer and System Engineering, Palaiseau, France \\ E-mail: \{moad.kissai, bruno.monsuez, adriana.tapus\}@ensta-paristech.fr \\ Didier Martinez \\ Groupe Renault / Chassis Systems Department, Guyancourt, France \\ didier.d.martinez@renault.com
}

\begin{abstract}
Most of automotive researches are focused on autonomous vehicles. Both industrials and academics are working on several changes, and the way to coordinate the increasing number of chassis systems is one among them. This paper proposes a more suitable control architecture for autonomous vehicles complexity. This architecture is based on control allocation techniques developed especially to handle over-actuated systems. The allocation is made by taking into account the tire dynamics couplings through a new linear tire model with varying parameters. Simulations of combined manoeuvres proved the potential of this architecture and its relevance for future autonomous vehicles.
\end{abstract}

Index Terms-Control allocation, chassis systems coordination, tire modelling, vehicle dynamics control, active rear wheel steering, autonomous vehicles.

\section{INTRODUCTION}

Vehicle handling and security have always been the main concern of car manufacturers and suppliers. Before the 1970's, passive safety systems were sufficient to ensure passengers' security. With the increasing number of vehicles on the roads, more complex situations have arisen, e.g., obstacle avoidance and sudden changes in surface conditions. Active safety systems were introduced late 1970's [1]. These systems are intended to help the driver control its vehicle when complex situations are encountered. An overview of the Advanced Driver Assistance Systems (ADAS) proposed in the last three decades is shown in Fig. 1.

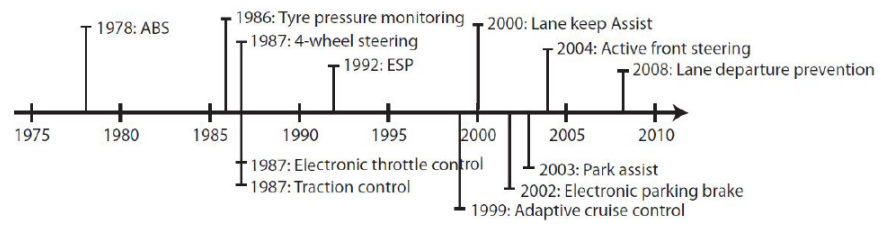

Fig. 1. Timeline of active safety systems introduction in passenger cars [2].

These systems have been introduced gradually as a luxury option to become a standard in all passenger cars. A fully autonomous system is thus a natural evolution towards safer and more comfortable vehicles. Here, the control is brought to the vehicle's center of gravity. The objective is the whole vehicle motion control. In Fig. 1, we can see that integrated systems involve only a single criterion that can be considered as a subobjective. For example, the Electronic Stability Program (ESP) uses the brake to generate a yaw moment and stabilize the vehicle [3]. Several systems are needed in the same vehicle to ensure autonomous driving. This has already began as today's passenger vehicles integrate more than a single chassis systems, making them overactuated systems [4]. Because these subsystems act on the same vehicle, interactions arise. The complexity of these interactions increases as the number of subsystems increases. Until now, the number of subsystems has been relatively low. Car manufacturers integrate chassis systems provided most of the time by suppliers. They then add few coordination strategies downstream these subsystems to avoid the possible conflicts that could arise from the subsystems interactions. These rules or prioritizing strategies are based on a deep understanding of the vehicle dynamics. The automotive engineer has to foresee the possible conflicting scenarios, and then elaborate the strategies that overcome them. As long as a low number of chassis systems is concerned, downstream coordination is sufficient to handle most conflicting situations [1]. However, as we have mentioned, the more we get close to autonomous driving, the more numerous chassis systems become, and the higher complexity gets. The automotive engineer will no longer be able to imagine all the possible scenarios and ensure vehicle safe operations. Therefore, the dynamic couplings should be mathematically described in order to find a solution. Moreover, a search towards an optimum should be conducted. This is important for over-actuated systems where various solutions can be found. In fact, if the control problem presents various solutions, secondary objectives could be fulfilled by choosing the right cost function to minimize [5]. Not only conflicts could be handled, but also better performances could be achieved. That implies a different way to act on chassis systems. To do so, different inputs should be provided to chassis systems. Coordination is then made upstream the subsystems. Unlike the downstream approach, the conflicts are prevented rather than mitigated. A larger comparison between both downstream and upstream approaches is provided in [6]. The upstream approach takes account of the dynamic couplings to find the best commands distribution. These couplings 
are generally non-linear. Recently, a new linear tire model with varying parameters has been proposed to depict tire combined dynamics while keeping its linearity [7].

This paper has a dual purpose. First, the downstream coordination and upstream distribution architectures are compared. This comparison is conducted to prove the potential of the upstream approach to handle subsystems interactions. A relatively simple example about today's vehicles is considered. A passenger car equipped with an Active Rear Steering (ARS) and Braking-Based Vehicle Dynamics Control (VDC) is studied using both approaches. Second, the importance of using the new linear tire model with varying parameters to enable realistic and safe commands distribution is demonstrated. Matlab/Simulink ${ }^{\circledR}$ has been used to simulate different realistic scenarios. Results showed better performances in case of the upstream approach using a basic control allocation technique and the new tire model, while the downstream approach could jeopardize vehicle operations as it does not take into account the dynamic couplings.

This paper is structured as follows: We start in Section II by presenting the currently used downstream coordination architecture. Section III presents the future upstream distribution architecture, and the new tire model that enables better control allocation. Different scenarios are simulated using Matlab/Simulink ${ }^{\circledR}$, and results are compared in Section IV. A discussion about the relevance of both the upstream approach along with the new tire model is provided in Section V. Conclusions and future works are outlined in Section VI.

\section{Downstream COORDination APPROACH}

For autonomous vehicles, both longitudinal and lateral performances should be met using a virtual pilot. Combined behaviour is then the most interesting case to study within this framework. Therefore, Active Rear Steering and the brakingbased Vehicle Dynamics Control have been chosen to be integrated in the same vehicle. Several today's passenger cars are equipped with both systems, e.g., the Renault Talisman [8]. Rear wheels can be steered and reach $\pm 5^{\circ}$ at most. Pressure commands for the VDC are limited to $[0,200]$ bar.

\section{A. Control Architecture}

As chassis systems are provided by different suppliers, they are often designed without taking into account the other subsystems. Inputs to these subsystems are then a physical quantity reference related directly the the vehicle itself. Their outputs are commands to the related actuator. The chassis system internal working is usually provided as a black box. With this configuration, the automotive engineer can only act downstream the subsystems, at their outputs. Fig. 2 illustrates this approach in the context of our example.

\section{B. System Modelling}

As only the yaw rate is concerned, one common approach is to use the bicycle model [9]. only lateral forces are taken into account. Because our goal is to first represent today's situation, this model is chosen in the context of the downstream

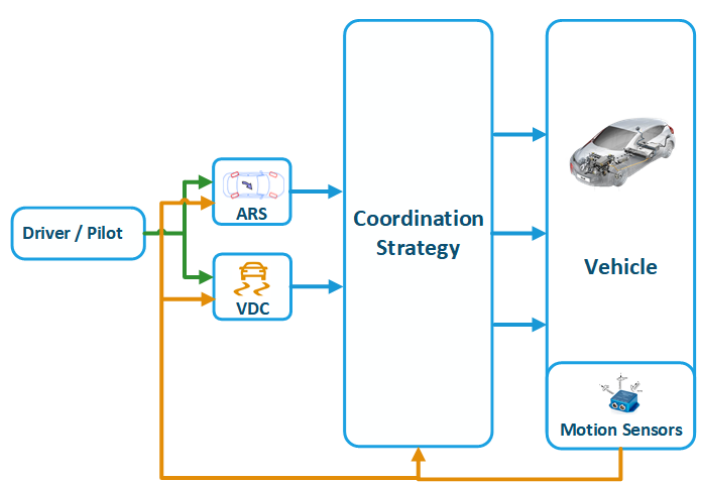

Fig. 2. Structure of the downstream coordination approach (adapted from [6])

approach. The linear tire model used in this case is expressed as follows [1],[4]:

$$
\left\{\begin{array}{l}
F_{y_{f}}=C_{\alpha_{f}} \alpha_{f} \\
F_{y_{r}}=C_{\alpha_{r}} \alpha_{r}
\end{array}\right.
$$

With:

- $F_{y_{f}}$ and $F_{y_{r}}$ : the lateral force at the front axle and the lateral force at the rear axle, respectively,

- $C_{\alpha_{f}}$ and $C_{\alpha_{r}}$ : the front equivalent cornering stiffness and the rear equivalent cornering stiffness, respectively,

- $\alpha_{f}$ and $\alpha_{r} \quad$ : the front equivalent side-slip and the rear equivalent side-slip, respectively.

Where:

$$
\left\{\begin{array}{l}
\alpha_{f}=\delta_{f}-\frac{V_{y}+\dot{\psi} l_{f}}{V_{x}} \\
\alpha_{r}=\delta_{r}-\frac{V_{y}-\dot{\psi} l_{r}}{V_{x}}
\end{array}\right.
$$

With:

- $\delta_{f}$ and $\delta_{r}$ : the front equivalent wheel angle and the rear equivalent wheel angle, respectively,

- $l_{f}$ and $l_{r}$ : the distance between the front axle and the vehicle's centre of gravity and the distance between the rear axle and the vehicle's centre of gravity, respectively,

- $V_{x} \quad$ : longitudinal velocity of the vehicle,

- $V_{y} \quad$ : lateral velocity of the vehicle,

- $\dot{\psi} \quad$ : yaw rate of the vehicle.

By using simplified Newton's laws of motion, we find:

$$
\left\{\begin{aligned}
M\left(\dot{\psi} V_{x}+s V_{y}\right) & =F_{y_{f}}+F_{y_{r}} \\
J_{z} s \dot{\psi} & =F_{y_{f}} l_{f}-F_{y_{r}} l_{r}+M_{v d c}
\end{aligned}\right.
$$

With:

- $M \quad$ : vehicle's mass,

- $J_{z} \quad$ : vehicle's yaw moment of inertia,

- $M_{v d c}$ : yaw moment generated by the VDC system,

- $s \quad$ : Laplace operator.

By using equations (1)-(6) with $V_{y}, \dot{\psi}, F_{y_{f}}, F_{y_{r}}, \alpha_{f}$, and $\alpha_{r}$ as unknown variables, transfer functions from $\delta_{f}, \delta_{r}$, and $M_{v d c}$ to $\dot{\psi}$ can be expressed. 


\section{Control Synthesis}

In our case, three commands can influence the yaw rate: the front steering $\delta_{f}$, the rear steering $\delta_{r}$, and the differential braking $M_{v d c}$. Only rear steering (ARS) and differential braking (VDC) are considered in control synthesis. The front steering is directly transmitted to the wheels and used to determine the driver desire, and therefore the yaw rate reference.

1) Yaw rate reference: As the yaw rate reference is determined from the driver command, the transfer function from the front steer to the yaw rate is used. Moreover, to ensure fast computation of the reference, only the steady-state is considered $(s=0)$ [1].

2) Coordination strategy: Because of the very simplified linear tire model and the negligence of combined slip, this latter should be avoided. This means that the ARS and the VDC should operate at the same time only if necessary ${ }^{1}$. One system should be then prioritized over the others. A preliminary comparison study is then required to establish the priority strategy. However, the ARS generates a steering angle, while the VDC generates a yaw moment. Either the yaw moment has to be converted to an equivalent angle or the rear angle to a yaw moment. Because we already know the maximum angle that could be generated by the ARS $\left( \pm 5^{\circ}\right)$, we choose to convert the VDC yaw moment to an equivalent angle. We obtain the graph in Fig. 3.

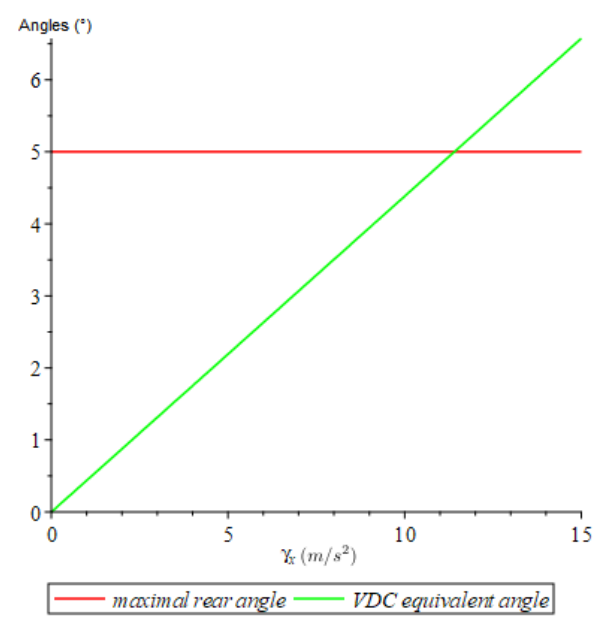

Fig. 3. Comparison of 4RS and VDC angle commands

Consequently, a deceleration of almost $11.4 \mathrm{~m} / \mathrm{s}^{2}$ is needed in order to exceed the maximal angle provided by the ARS. The VDC should not be prioritized unless the deceleration exceeds $11.4 \mathrm{~m} / \mathrm{s}^{2}$.

3) Subsystems controllers: As the subsystems are located downstream the coordination layer (see Fig. 2), their controllers are based on transfer functions from system angle command to directly the vehicle's yaw rate. A gain-scheduled $\mathrm{PI}^{2}$ has been chosen for both ARS and VDC systems. The

\footnotetext{
${ }^{1}$ When one system fails or saturates

${ }^{2}$ The longitudinal velocity is the scheduling parameter.
}

detailed design of these controllers goes beyon the scope of this paper.

\section{UPSTREAM DistribUtion APPROACH}

Coordinating the chassis systems downstream their controllers implies that the nature of conflicts is predictable. However, one cannot foresee all the possible scenarios. An alternative approach is an upstream distribution of commands. Here, the combined dynamics should be formalized in order to distribute well the commands. Particularly, the tire model should be able to take into account the combined slip as it is the sole effector of the vehicle. Hence, the relevance of the new linear tire model with varying parameters [7]. This model makes it possible to elaborate a better commands distribution by taking into account a more realistic tire potential.

\section{A. Control Architecture}

The layer to be added in order to synergize chassis systems is located now upstream these subsystems. Fig. 4 illustrates this concept.

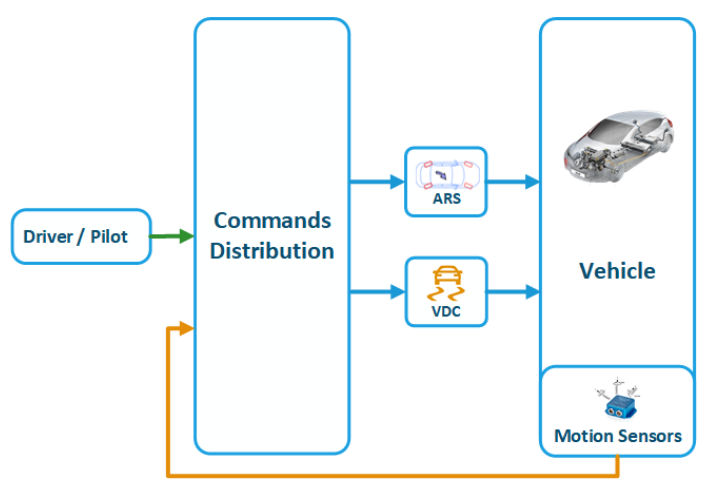

Fig. 4. Structure of the upstream distribution approach (adapted from [6]

\section{B. System Modelling}

The idea is to calculate first the necessary forces to move the vehicle applied at its centre of gravity, and then optimally distribute these forces to the four tires depending on their potentials. Two subsystems are available to control the yaw rate. The overall system is then over-actuated. Control allocation techniques were developed especially for this category of problems [4]. The control allocation problem can be defined as follows [10]: find the control vector, $\vec{\delta} \in \mathbb{R}^{n}$ such that

$$
\mathbf{B} \vec{\delta}=\vec{d}_{d e s}
$$

subject to

$$
\left\{\begin{aligned}
& \vec{\delta}_{\min } \leq \vec{\delta} \leq \vec{\delta}_{\max } \\
& \dot{\vec{\delta}} \leq \dot{\vec{\delta}}_{\max }
\end{aligned}\right.
$$

where $\mathbf{B} \in \mathbb{R}^{n \times m}$ is a control effectiveness matrix, $\vec{\delta}_{\text {min }} \in \mathbb{R}^{n}$ and $\vec{\delta}_{\text {max }} \in \mathbb{R}^{n}$ are the lower and upper position limits, respectively, $\dot{\vec{\delta}} \in \mathbb{R}^{n}$ is the control rate, $\overrightarrow{\vec{\delta}}_{\text {max }} \in \mathbb{R}^{n}$ is the maximum control rate, $\vec{d}_{\text {des }} \in \mathbb{R}^{m}$ are the desired accelerations, $n$ is the number of control effectors, and $m$ is the number of axes to 
control $(n>m)$. Different techniques can be used in order to synthesis $\vec{\delta}$. A large overview can be found in [5],[10],[11].

1) Tire forces: Unlike the downstream coordination strategy presented in this paper, the upstream approach using daisychain method could make use of both ARS and VDC at the same time (see Section IV). The ARS system generates lateral tire forces, while the VDC system generates longitudinal tire forces. The overall adhesion is delimited by a "friction ellipse" [12]. Tire potential is delimited by the product $\mu F_{z}$, where $\mu$ is the coefficient of friction that represents the quality of the interface rubber/ground, and $F_{z}$ is the vertical load. Forces' saturation can be represented as follows [7]:

$$
\left\{\begin{array}{l}
F_{x} \leq \sqrt{\left(\mu F_{z}\right)^{2}-F_{y}^{2}} \\
F_{y} \leq \sqrt{\left(\mu F_{z}\right)^{2}-F_{x}^{2}}
\end{array}\right.
$$

As long as control synthesis is concerned, a linear model is prefered. This mothivates the use a linear tire model with varying parameters [6]. This model is expressed as follows [7]:

$$
\left\{\begin{array}{l}
F_{x}=C_{s}^{*}\left(\alpha, \mu, F_{z}\right) \kappa \\
F_{y}=C_{\alpha}^{*}\left(\kappa, \mu, F_{z}\right) \alpha
\end{array}\right.
$$

where:

- $\kappa \quad:$ the longitudinal slip,

- $C_{s}^{*}\left(\alpha, \mu, F_{z}\right)$ : the tire varying longitudinal stiffness with respect to the side-slip $\alpha$, the friction coefficient $\mu$, and the vertical load $F_{z}$,

- $C_{\alpha}^{*}\left(\kappa, \mu, F_{z}\right)$ : the tire varying cornering stiffness with respect to the longitudinal slip $\kappa, \mu$, and $F_{z}$.

Detailed expressions of the varying stiffness and their development are provided in [6].

Moreover, as the ARS system generates the same steering angle in both tires, only the lateral force at the rear axle level is taken account of. Unlike the ARS, the VDC has the ability to generate different longitudinal forces at each tire. The control vector is then:

$$
\vec{\delta}=\left[\begin{array}{c}
F_{x_{f l}} \\
F_{x_{f r}} \\
F_{x_{r l}} \\
F_{x_{r r}} \\
F_{y_{r}}
\end{array}\right]
$$

Therefore, assuming that the vertical load and friction coefficient can be different at the level of each tire, control vector limits can be expressed as follows:

$$
\vec{\delta}_{\text {max }}=\left[\begin{array}{c}
\sqrt{\left(\mu_{f l} F_{z_{f l}}\right)^{2}-F_{y_{f l}}^{2}} \\
\sqrt{\left(\mu_{f r} F_{z_{f r}}\right)^{2}-F_{y_{f r}}^{2}} \\
\sqrt{\left(\mu_{r l} F_{z_{r l}}\right)^{2}-F_{y_{r l}}^{2}} \\
\sqrt{\left(\mu_{r r} F_{z_{r r}}\right)^{2}-F_{y_{r r}}^{2}} \\
\sqrt{\left(\mu_{r} F_{z_{r}}\right)^{2}-F_{x_{r}}^{2}}
\end{array}\right]
$$

The same goes for $\vec{\delta}_{\text {min }}$ with a minus sign in each component.
The vertical load at the level of each tire can be defined as [4]:

$$
\left\{\begin{array}{l}
F_{z_{f l}}=\frac{1}{2} M g \frac{l_{r}}{L}-\frac{1}{2} M a_{x} \frac{h}{L}-\frac{1}{2} M a_{y} \frac{h}{E} \\
F_{z_{f r}}=\frac{1}{2} M g \frac{l_{r}}{L}-\frac{1}{2} M a_{x} \frac{h}{L}+\frac{1}{2} M a_{y} \frac{h}{E} \\
F_{z_{r l}}=\frac{1}{2} M g \frac{l_{r}}{L}+\frac{1}{2} M a_{x} \frac{h}{L}-\frac{1}{2} M a_{y} \frac{h}{E} \\
F_{z_{r r}}=\frac{1}{2} M g \frac{l_{r}}{L}+\frac{1}{2} M a_{x} \frac{h}{L}+\frac{1}{2} M a_{y} \frac{h}{E}
\end{array}\right.
$$

where:

- $g$ : gravitational acceleration,

- $a_{x}$ : vehicle's longitudinal acceleration,

- $a_{y}$ : vehicle's lateral acceleration,

- $h$ : height of vehicle's centre of gravity.

Here, we suppose that $\mu_{i j}$ can be estimated [13].

2) Generalized forces calculation: We recall that the main objective is yaw rate control. As long as the brake is concerned, the longitudinal velocity could be penalised. Control allocation can be also used to mitigate the influence on longitudinal speed. Two generalised equations are then considered:

$$
\left\{\begin{aligned}
M s V_{x} & =F_{x_{t o t}} \\
J_{z} s \dot{\psi} & =M_{z_{t o t}}
\end{aligned}\right.
$$

where:

- $F_{x_{t o t}}$ : the total longitudinal force required at the vehicle's centre of gravity,

- $M_{z_{\text {tot }}}$ : the total yaw moment required at the vehicle's centre of gravity.

In this case, we have:

$$
\vec{d}_{d e s}=\left[\begin{array}{c}
F_{x_{t o t}} \\
M_{z_{t o t}}
\end{array}\right]
$$

Because of the differential nature of equations (20) and (21), a high-level controller is first required to compute $\vec{d}_{d e s}$.

3) Effectiveness matrix: To compute control commands to the four wheels, a four-wheeled planar vehicle model is necessary. Considering only controllable tire forces with $\delta_{r} \ll$, we find:

$$
\left\{\begin{aligned}
F_{x_{t o t}}= & \left(F_{x_{f l}}+F_{x_{f r}}\right) \cos \left(\delta_{f}\right)+F_{x_{r l}}+F_{x_{r r}} \\
M_{z_{t o t}}= & \left(F_{x_{f l}}+F_{x_{f r}}\right) l_{f} \sin \left(\delta_{f}\right) \\
& +\left(F_{x_{f r}}-F_{x_{f l}}\right) \frac{E}{2} \cos \left(\delta_{f}\right) \\
& +\left(F_{x_{r r}}-F_{x_{r l}}\right) \frac{E}{2}-F_{y_{r}} l_{r}
\end{aligned}\right.
$$

with $F_{y_{r}}=F_{y_{r l}}+F_{y_{r r}}$. From equations (7),(14),(22), $(23),(24)$, we get the following effectiveness matrix expression:

$$
\mathbf{B}=\left[\begin{array}{ccccc}
\cos \left(\delta_{f}\right) & \cos \left(\delta_{f}\right) & 1 & 1 & 0 \\
b_{2,1} & b_{2,2} & -\frac{E}{2} & \frac{E}{2} & -l_{r}
\end{array}\right]
$$

where:

- $b_{2,1}=l_{f} \sin \left(\delta_{f}\right)-\frac{E}{2} \cos \left(\delta_{f}\right)$, 
- $b_{2,2}=l_{f} \sin \left(\delta_{f}\right)+\frac{E}{2} \cos \left(\delta_{f}\right)$.

\section{Control Synthesis}

Control allocation in this case consists on adding a new abstraction level. Rather than calculating actuator commands directly from desired velocities, we add effector constraints in the middle of the control chain. A multi-layer architecture is then required [6].

1) High-level controller: Here, we consider equations (20) and (21). Consequently, two simple PI controllers are designed to meet high-level control requests.

2) Control allocation strategy: To provide a fair comparison between downstream and upstream approaches, a prioritization of the ARS with respect to the VDC is also privileged. However, distribution of commands is not the same as we take into account the tires potential. To do so, two control allocation methods are used: daisy-chain method to prioritize the ARS, and weighted pseudo-inverse method to distribute force requests depending on tires potential.

\section{- Daisy-chain}

It is about forcing an hierarchy for control effectors. The difference with respect to the downstream approach is the condition to move to the second subsystem. Here, actuator saturation or tire saturation is the switching condition. In addition, when a control saturates, an error between the desired forces and those generated by control effectors arises. Only this error is transmitted to the second set of subsystems. Therefore, the VDC only activates when the rear lateral force saturates or the rear steering angle reaches $\pm 5^{\circ}$. The control allocation problem is then separated into two sub-problems. The first set contains only the ARS system. As only rear steering can be generated, only yaw moment is considered. Longitudinal force is ignored. We have then:

$$
F_{y_{r}}=-\frac{1}{l_{r}} M_{z},
$$

When ARS is saturated ${ }^{3}$, the deficiency is transmitted to the VDC system. The second sub-problem is then defined as:

$$
\left[\begin{array}{c}
F_{x_{t o t}} \\
M_{z}+l_{r} F_{y_{r}}
\end{array}\right]=\mathbf{B}_{2}\left[\begin{array}{c}
F_{x_{f l}} \\
F_{x_{f r}} \\
F_{x_{r l}} \\
F_{x_{r r}}
\end{array}\right]
$$

where $\mathbf{B}_{\mathbf{2}}=\left[\begin{array}{cccc}\cos \left(\delta_{f}\right) & \cos \left(\delta_{f}\right) & 1 & 1 \\ b_{2,1} & b_{2,2} & -\frac{E}{2} & \frac{E}{2}\end{array}\right] . \mathbf{B}_{\mathbf{2}}$ has to be inverted. However, it is not a square matrix [11]. A pseudoinverse can still be provided. Moreover, to favour one control command over another depending on tire potential, weighting matrices could be used [4]. Therefore, a "weighted pseudoinverse" is used for brake forces allocation.

- Weighted pseudo-inverse

\footnotetext{
${ }^{3}$ The cause could be tire saturation or actuator saturation.
}

It is an optimization technique based on a pseudo inversion of the non-square matrix $\mathbf{B}$. The problem is formulated as follows [11]:

$$
\min _{\vec{\delta}} J=\min _{\vec{\delta}} \frac{1}{2}(\vec{\delta}+\vec{c})^{T} \mathbf{W}(\vec{\delta}+\vec{c})
$$

subject to (7), where $\mathbf{W} \in \mathbb{R}^{n \times n}$ is a weighting matrix and $\vec{c} \in \mathbb{R}^{n}$ is an offset vector used to represent an off-nominal condition. The resolution method using the Hamiltonian can be found in [11]. The weighting matrix can be adjusted to favor one effector over another. This is done by increasing the weight of the less attractive actuator. Therefore, to take into account the tire potential, we introduce the inverse of the tire force saturation in the weighting matrix diagonal. When approaching saturation, the weight denominator approaches to 0 and therefore, the weight approaches to $\infty$. The weighting matrix chosen is expressed as:

$$
\mathbf{W}=\left[\begin{array}{cccc}
w_{f l} & 0 & 0 & 0 \\
0 & w_{f r} & 0 & 0 \\
0 & 0 & w_{r l} & 0 \\
0 & 0 & 0 & w_{r r}
\end{array}\right]
$$

where $w_{i j}=\frac{1}{\sqrt{\left(\mu_{i j} F_{z_{i j}}\right)^{2}-F_{y_{i j}}^{2}}}$.

3) Low-level controllers: Two major criteria have to be considered in controller design for this case. First, the lowlevel layer is located in the most inner loop. It should be much faster in order not to influence the high-level control. The crossover frequency of the low-level control is chosen to be 10 times higher than the high-level's. Moreover, the same crossover frequency is chosen for rear steering angle command and braking torques commands. Reallocation should be caused by tire or actuator saturations and not by one of the controllers delay with respect to the others. Secondly, no overshoot should be allowed. Control allocation is made by taking into account the tire saturation. At hazardous situations, it could allocate the maximum allowable tire force. This force must not be exceeded, otherwise the vehicle could loose its stability.

Here, transfer function from tire forces the rear steering angle and brake torques are considered. Simple feed-forward PI controllers are designed. Again, details of this design go beyond the scope of this paper.

\section{COMPARISON OF COORDINATION APPROACHES}

In order to consolidate our hypothesis, simulations using Matlab/Simulink ${ }^{\circledR}$ are carried out. Both approaches are programmed in Simulink platform to enable their comparison. A slalom maneuver is carried out with a longitudinal speed of $100 \mathrm{~km} / \mathrm{h}$. At time $t=4 \mathrm{~s}$, we block the ARS system to test the system safety. Fig. 5 illustrates this failure. As no overshoot is allowed, the upstream approach generates a rear angle smaller than the downstream approach. Regarding yaw rate, Fig. 6 illustrates the reconfiguration ability of each approach. The upstream approach exhibits a more important overshoot after the failure. This is mainly due to the activation of brake forces as Fig. 7 shows. As neither longitudinal acceleration does not 


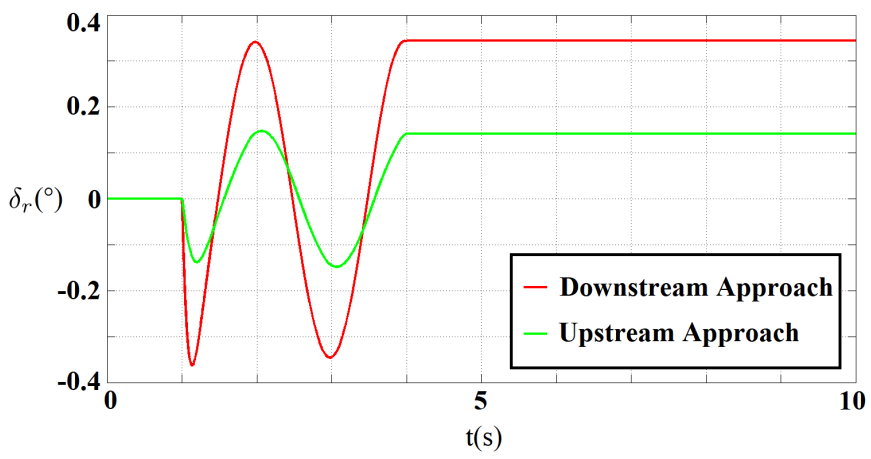

Fig. 5. Rear steering angle failure

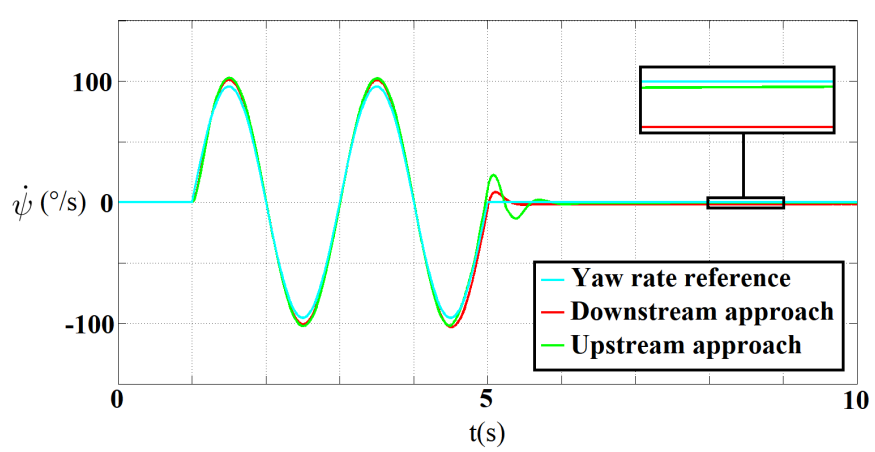

Fig. 6. Yaw rate at rear steering angle failure

exceed $11.4 \mathrm{~m} / \mathrm{s}^{2}$ nor rear angle actuator saturates, the VDC system does not activate in the downstream approach. No important overshoot is experienced in that case, but a steadystate yaw rate error remains. To keep the vehicle in a straight line, the driver has to continually steer the front wheels. This is not suitable for autonomous driving.

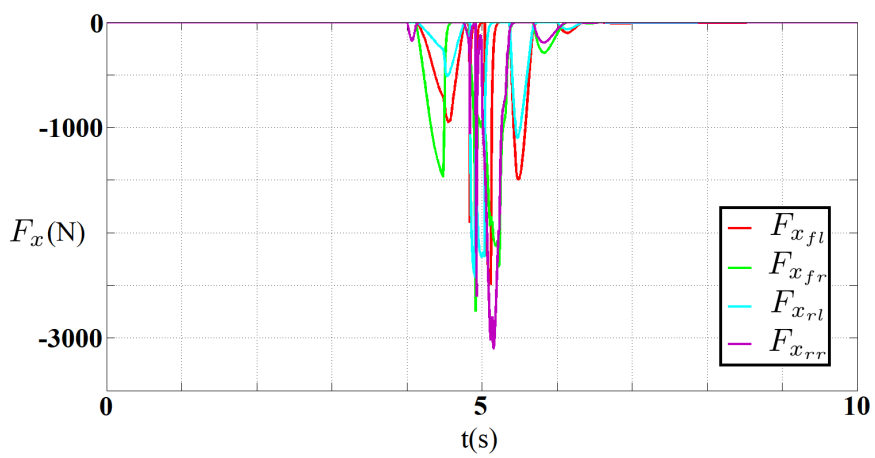

Fig. 7. Brake forces for the upstream approach in case of ARS failure

Regarding brake forces, when left brake forces are activated right ones do not and vice-versa to create a maximum yaw moment. However, for tires in the same side, there exists a slight difference between activated brake forces. This is caused by the difference in vertical loads at each tire which gives different potentials (Fig. 8). Thanks to the new tire model, tires with bigger potential are prioritized which gives better control allocation.

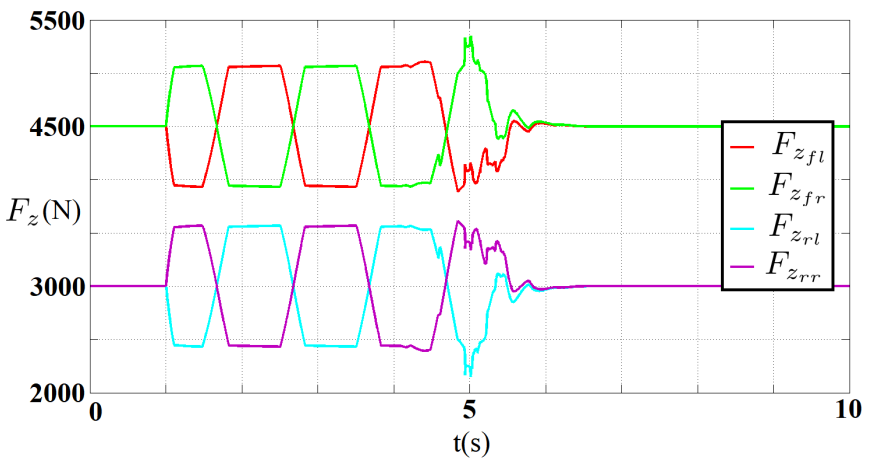

Fig. 8. Vertical loads taken account of in upstream approach.

Another objective is fulfilled by the upstream approach, which is longitudinal speed influence mitigation. In fact, as Fig. 9 shows, brake forces are chosen in a way to influence less the longitudinal speed and track again the reference as soon as possible.

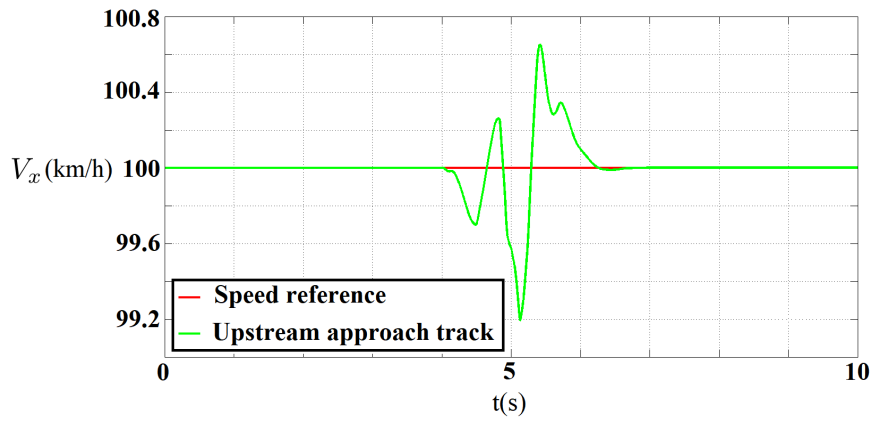

Fig. 9. Longitudinal speed tracking in upstream approach

\section{RELEVANCE OF THE UPSTREAM DISTRIBUTION APPROACH}

As we have previously mentioned, the number of integrated chassis systems is increasing. From Advanced Driver Assistance Systems (ADAS) to full autonomous driving, additional chassis systems are intended to be added to fulfil all driver manoeuvres. Both longitudinal and lateral control have to be ensured at the same time. Dynamic couplings can no longer be ignored. More complex modelling is needed, and therefore more complex control strategies are required.

\section{A. Necessity for an upstream distribution}

First, let us enumerate the advantages of the downstream approach that kept automotive manufacturers from substituting control architectures. From an industrial point of view, cost is always a major key. Considering Fig. 2 and the fact that chassis systems are developed individually, these latter are integrated without bringing any modification. No additional controllers are needed. Relatively simple coordination strategies are added downstream the subsystems to handle their interactions. This gives the opportunity for car manufacturers to proceed to bulk purchasing from their suppliers and take advantage from 
the economy of scale. Unfortunately, regarding the upstream approach, as we have demonstrated in Section III, additional high-level controllers are needed. Moreover, as inputs change, suppliers' controllers have to be modified. This architecture may also require additional sensors or estimators [14], especially for friction coefficient estimation. Nevertheless, as it is pointed out in [1], adding a high-level controller allows exploiting the same information in several subsystems. The number of sensors can then be reduced as well as computational overheads by not duplicating controller computations.

Another advantage for the downstream approach is its simplicity. For example, as it is described in Section II, only a preliminary study of chassis systems' influence on vehicle dynamics is needed so as to deduce which subsystem should be prioritized. In contrast, for control allocation, the problem should be mathematically described. Additional complex scenarios are then implicitly considered. A general solution to all possible scenarios should be provided. Dynamic couplings at the vehicle level and at the tire level should be considered. This makes the control synthesis more tedious.

In the context of autonomous driving, dynamic couplings have to be considered. Until now, the driver ensures longitudinal control by accelerating or braking. Most of ADAS are designed to stabilize the vehicle laterally. While controlling longitudinally the vehicle, the driver handles unconsciously the dynamic couplings. The virtual pilot has to take over and control the vehicle both longitudinally and laterally. A larger operation range is solicited in this case. More complex vehicle and tire modelling are required. As chassis systems are developed individually, they are generally based on different vehicle models [6]. Their integration is not necessary uniform. Rule-based strategies can handle the problem only if most of the scenarios can be predicted. The more we approach to autonomous driving, the more numerous are chassis systems to be added in the same vehicle to replace the driver, and the more numerous are the scenarios that cannot be predicted in advance. Therefore, the upstream approach would become necessary despite its complexity.

Control allocation methods express the problem as an optimization problem for an over-actuated system. This gives numerous solutions. Secondary objectives can be then satisfied. For example, the solution that consumes less energy or that gives more comfort can be favored. The upstream approach can then transform the problem in an opportunity. Choice of objectives and a supervisory (high-level control) strategy gives the possibility for car manufacturers to tune their vehicles behaviors. This is crucial for companies brand image.

\section{B. Necessity for a more accurate tire model}

As we have previously mentioned, going towards autonomous driving implies switching from a downstream coordination approach to an upstream distribution one. As tires are the sole effector for ground vehicles, commands distribution concerns more the four tires. Tire with more potential to influence vehicle dynamics should be favoured. This potential depends on mainly three criteria: combined slip, vertical load, and the interface rubber/ground state, which is represented by the friction coefficient. Not taking into account this criteria could saturate one or several tires and therefore destabilize the vehicle while other tires could be used to achieve the manoeuvre.

Tire potential manifests itself in its ability to adhere to the road. This could be represented by the tire stiffness. This stiffness should therefore be updated on-line to take into account the varying criteria mentioned. Moreover, the tire model should be suitable for control synthesis. Its complexity should be then reduced. A linear model is thus preferred. For all these reasons, the new linear tire model with varying parameters is more suitable for global chassis control synthesis, and should be therefore implemented in autonomous vehicles.

\section{CONCLUSION}

Two chassis systems coordination approaches have been compared in this paper. The downstream approach uses rulebased algorithms to favour one system over another, which makes it relatively simple. The upstream approach uses optimization-based algorithms to find the best solution for a single or multi-objectives control problem. Control allocation methods are designed especially to handle over-actuated systems. These methods are more complex with respect to rulebased algorithms. A vehicle equipped with an Active Rear Steering (ARS) system and a brake-based Vehicle Dynamics Control (VDC) system has been chosen to study chassis systems interactions as both influence yaw rate dynamics. Both architectures exhibit good yaw rate reference track. However, the upstream approach generates more realistic commands as a middle layer is added to take into account the tire dynamic couplings and saturation thanks to a new linear tire model with varying parameters.

However, it should be noted that in this paper, basic control allocation techniques have been used. The main goal is to compare coordination approaches through a simple example. More complex but more efficient control techniques are being tested. We expect that these techniques could handle more complex problems where more than two chassis systems are involved. More interesting scenarios could be handled as dynamic reconfiguration to handle switch issues as it was seen in the ARS failure manoeuvre, the $\mu$-split manoeuvre to benefit more from the opportunities that gives the new tire model, and so on.

Moreover, different vehicle models have been used in this paper. Results from downstream coordination could be overestimated as it uses a simple bicycle model. As a future work, a more complete vehicle model with different chassis systems is being developed in AMESim ${ }^{(c)}$ platform. This will provide a common platform to enable effective comparison of different algorithms. Experimentations are intended to be conducted with the collaboration of Renault in order to prove our claims. More attractive chassis could then be added gradually as the Four Wheel Drive (4WD) system or the Torque Vectoring (TV). We expect that with adding more chassis systems, the 
downstream approach can no longer handle their interactions, and the upstream approach would become more pertinent.

\section{ACKNOWLEDGMENT}

The authors would like to thank Dr. Xavier Mouton from the Group Renault for his supervision of the Vehicle Motion Control (VMC) project.

\section{REFERENCES}

[1] M. A. Selby, "Intelligent Vehicle Motion Control," PhD thesis, University of Leeds, Feb. 2003.

[2] M. Gerard and E.Y. Lopes, "Global Chassis Control and Braking Control using Tyre Forces Measurement," TU Delft, 2011.

[3] E. Liebemann, K. Meder, J. Schuh, and G. Nenninger, "Safety and performance enhancement: The bosch Electronic Stability Control (ESP)," in SAE Technical Paper 2004-21-0060, 2004.

[4] A. Soltani, "Low Cost Integration of Electric Power-Assisted Steering (EPAS) with Enhanced Stability Program (ESP)," PhD thesis, Cranfield University, 2014.

[5] M. Bodson, "Evaluation of optimization methods for control allocation," in Journal of Guidance, Control, and Dynamics, vol. 25, no. 4, pp. 703711, 2002.

[6] M. Kissai, B. Monsuez and A. Tapus, "Review of integrated vehicle dynamics control architectures," in 2017 European Conference on Mobile Robots (ECMR), Paris, 2017, pp. 1-8

[7] M. Kissai, B. Monsuez, A. Tapus and D. Martinez, "A new linear tire model with varying parameters," in 2017 2nd IEEE International Conference on Intelligent Transportation Engineering (ICITE), Singapore, 2017, pp. 108-115.

[8] Moss and Darren, "Renault Talisman revealed," in autocar.co.uk, Haymarket Media Group, Retrieved 6 July 2015.

[9] E. Ono, K. Takanami, N. Iwama, Y. Hayashi, Y. Hirano, Y. Satoh, "Vehicle integrated control for steering and traction systems by $\mu$ synthesis", in Automatica, Vol. 30, Issue 11, pp. 1639-1647, 1994.

[10] T. A. Johansen and T. I. Fossen, "Control Allocation - A survey," in Automatica, Vol. 49, Issue 5, May 2013, pp. 10871103.

[11] M. W. Oppenheimer, D. B. Doman, and M. A. Bolender, "Control Allocation for Over-actuated Systems," in 2006 14th Mediterranean Conference on Control and Automation, Ancona, 2006, pp. 1-6.

[12] H.B. Pacejka, "Tyre and Vehicle Dynamics," Second Edition. Elsevier, Butterworth-Heinemann, 2006.

[13] J. Svendenius, "Tire Modeling and Friction Estimation, Department of Automatic Control, Lund University, 2007.

[14] L. Heidrich, B. Shyrokau, D. Savitski, V. Ivanov, K. Augsburg and D. Wang, "Hardware-in-the-loop test rig for integrated vehicle control systems," in Proc. 7th IFAC Symp. Adv. Automot. Control, Tokyo, Japan, 2013, pp. 683-688.

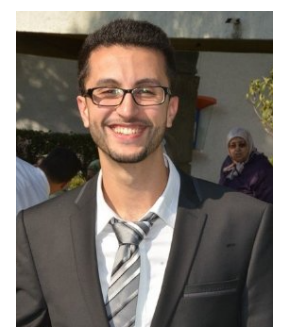

Moad Kissai was born in Rabat, Morocco, in 1992. $\mathrm{He}$ received his M.Sc. in mobility and electric vehicles from Arts et Métiers ParisTech, France in 2015, and his degree of Engineer in electromechanics from National Graduate Engineering School - Mines Rabat, Morocco in 2014.

$\mathrm{He}$ is currently pursuing the $\mathrm{PhD}$ degree on automotive and control engineering with the Computer Science and System Engineering Department (U2IS), at ENSTA ParisTech, France. His current research interests include vehicle motion control, chassis systems coordination, control allocation, and optimal control.

Mr. Kissai is an active Student Member of IEEE Control Systems, Robotics and Automation Societies.

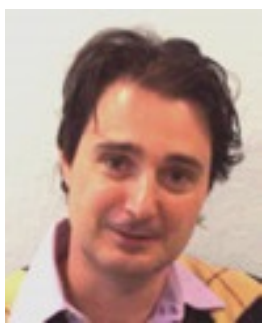

Bruno Monsuez received his $\mathrm{PhD}$ in computer science in 1994, and his degree of Engineer in 1989 from École Polytechnique, France.

He started his academic career at École Normale Supérieure, Paris, France working on automated verification of Software and Hardware Systems. He was on leave about 10 years working for pioneering the formal system design methodology and formal verification methods in the industry. His current research interests are on the design and validation of highly automated systems as well as autonomous systems for safety critical systems. He is currently Full Professor in the Autonomous Systems and Robotics Lab in the Computer Science and System Engineering Department (U2IS), at ENSTA ParisTech, France.

Prof. Bruno Monsuez served on PCs and as PC chair for numerous international workshops and conferences. He is the steering committee chair of Int. Conference on Verification and Evaluation of Computer and Communication Systems (VECoS) and will coorganize with Sadek Bensalem the VECoS 2018 in Grenoble.

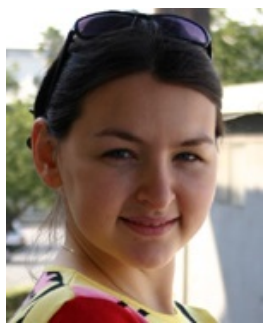

Adriana Tapus obtained the French Habilitation (HDR) for her thesis entitled "Towards Personalized Human-Robot Interaction" from Pierre and Marie Curie University (UPMC), France in 2011. She received her $\mathrm{PhD}$ in computer science from Swiss Federal Institute of Technology Lausanne (EPFL), Switzerland in 2005, and her degree of Engineer in computer science and engineering from Politehnica University of Bucharest, Romania in 2001.

She worked as an Associate Researcher at the University of Southern California (USC), where she was among the pioneers on the development of socially assistive robotics, also participating to activity in machine learning, human sensing, and human-robot interaction. Her main interests are on long-term learning (i.e. in particular in interaction with humans), human modeling, and on-line robot behavior adaptation to external environmental factors. She is currently a Full Professor in the Autonomous Systems and Robotics Lab in the Computer Science and System Engineering Department (U2IS), at ENSTA ParisTech, France.

Prof. Tapus is an Associate Editor for International Journal of Social Robotics (IJSR), ACM Transactions on Human-Robot Interaction (THRI), and IEEE Transactions on Cognitive and Developmental Systems (TCDS) and in the steering committee of several major robotics conferences (General Chair 2019 of HRI, Program Chair 2018 of HRI, General Chair 2017 of ECMR). She has more than 150 research publications and she received the Romanian Academy Award for her contributions in assistive robotics in 2010. She was elected in 2016 as one of the 25 women in robotics you need to know about. She's also the PI of various EU and French National research grants. Further details about her research and her activities can be found at http://www.enstaparistech.fr/ tapus.

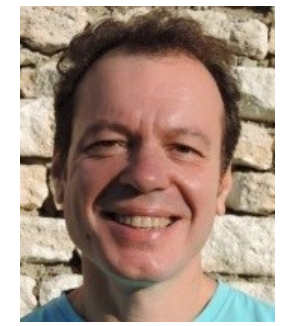

Didier Martinez received his $\mathrm{PhD}$ in automatic control from The University of Bordeaux 1, France in 1996.

He worked as an Automatic Control Engineer for EADS (now Airbus Aerospace) from 1996 to 2002. His work concerned flight control design and thrust vector control for Ariane 5 Launcher and M51 French Missile. In 2002, he started working for Groupe Renault as an automatic control engineer and technical leader regarding chassis systems (Electric Power-Assisted Steering, Vehicle Dynamics Control, 4-Wheel Steering, and 4-Wheel Drive). He is currently developing new vehicle motion control strategies using all subsystems above at the same time to optimize and control the chassis behavior depending on the driving context. 